\title{
APPLICATION OF THE ANALYTIC HIERARCHY PROCESS FOR CHOICE OF OPTIMAL SOFTWARE SYSTEM CONFIGURATION
}

\author{
Viatcheslav Miroshnikov, Alexandre Podvessovski \\ Bryansk State Technical University \\ 50-Let Oktyabrya boul. 7, 241035 Bryansk, Russia \\ alex@podv.bitmcnit.bryansk.su
}

Keywords: software system, configuration, the Analytic Hierarchy Process, the Resources Allocation Method, fuzzy interval

\begin{abstract}
Summary: The mathematical models and algorithms for choice of optimal software system configuration have been presented. The generalized choice algorithm includes three stages (and there are three mathematical models respectively): a class of system is chosen at the first stage, a type of system is chosen at the second stage, and a particular configuration is chosen at the third one. The Analytic Hierarchy Process (AHP) and the Resources Allocation methods including the AHP as one of their components, underlie the mentioned models. The model and algorithm for choice of class of system have been described for both crisp and fuzzy environments.
\end{abstract}

\section{Introduction}

Informatization is the most effective way for industrial development today. That means application of progressive information technologies for automation of design and manufacturing (CAD/CAM/CAE systems), production management (ERP/MRP systems), documents circulation (Workflow systems), etc. Today the software market presents a number of such systems, which differ according to functional possibilities, scale of tasks being solved, requirements to the hardware environment, costs and expenses for maintenance. Therefore, entering the way of informatization, an industrial plant often faces a problem of choosing such a software system and its configuration, which would most satisfy the specific problems of this plant, taking into consideration its financial possibilities. The importance of such a choice being right is predetermined first of all by the fact, that any mistake at this stage may cancel the whole effect of the system introduction, and this may cause significant financial loses for the plant, because of high cost of such systems (in the order of hundreds thousand USD).

A computational methodology for choice of optimal configuration for a software system of any class and type is presented in the paper. Using of this methodology would increase the scientific and technical validity of decisions being made by an industrial plant while applying of new information technologies, and also would make investments for these purposes less risky. The methodology is based on application of the AHP (Saaty, 1980) in combination with elements of fuzzy sets theory (Zadeh, 1973).

\section{The generalized algorithm for choice of optimal software system configuration}

The choosing process is carried out in the framework of such taxonomic hierarchical structure of software systems as "family-class - type-configuration". At the same time it is subject to the framework of a certain family of systems. In other words, configurations can be chosen among the family of either $\mathrm{CAD} / \mathrm{CAM} / \mathrm{CAE}$ or ERP/MRP or Workflow systems, etc. Next, for example the classes of systems from the CAD/CAM/CAE family are "heavy", "medium" and "light". The "heavy" CAD/CAM/CAE systems class contains such types of systems as CATIA, Pro/ENGINEER, UNIGRAPHICS, EUCLID, etc. The methodology being described implies the three-stages approach for the choosing process. A class of sys- 
tem is chosen at the first stage, a type of system is chosen at the second stage, and a particular configuration is chosen at the third one. This approach is shown in Fig. 1 as a generalized choice algorithm scheme.

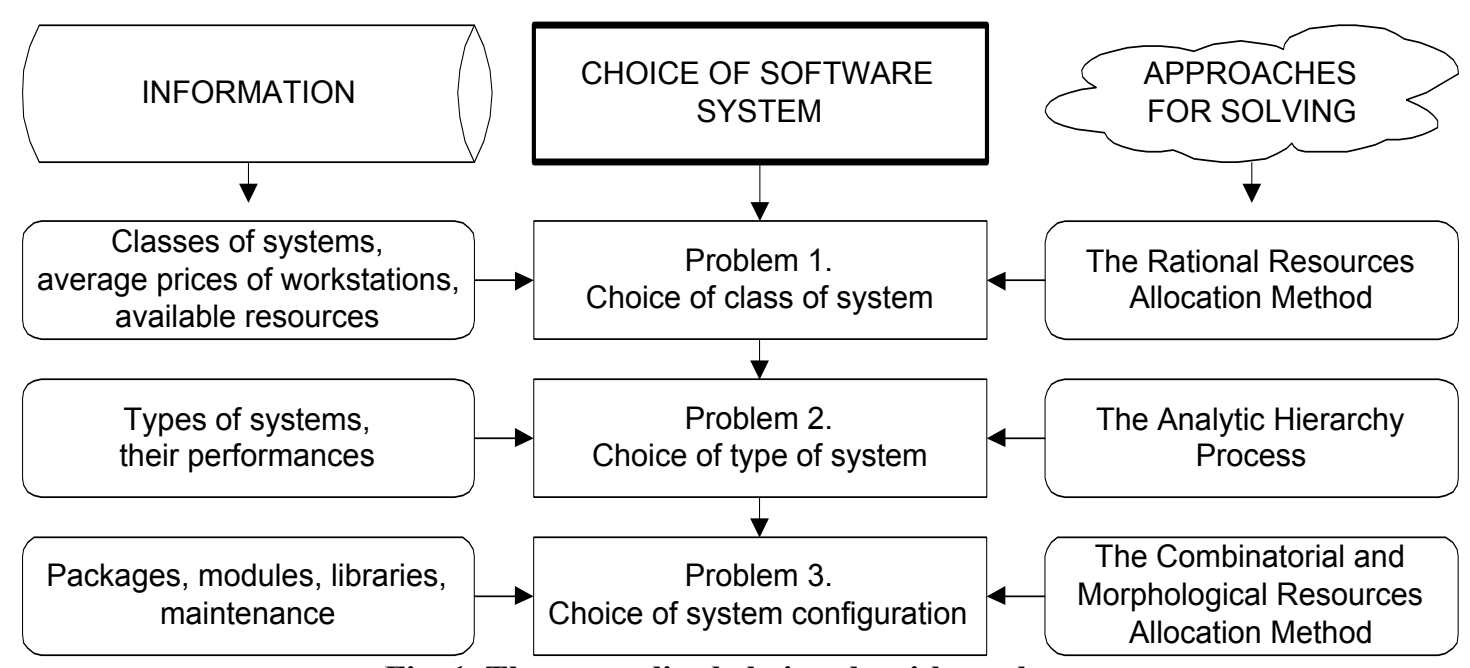

Fig. 1. The generalized choice algorithm scheme

The kind of informational environment (crisp or fuzzy) for the problem is taken into consideration in the frameworks of this algorithm. Therefore the descriptions of the particular stages are given separately for crisp and fuzzy cases.

\section{The choice of optimal decision in crisp environment}

\subsection{The choice of class of system}

The developed by the authors mathematical model for this stage is based on the method of Rational Resources Allocation (Andreichicov and Andreichicova, 2000). According to this model, the problem for choice of class of system can be formalized as follows. Let $A_{j}(j=1, \ldots, k)$ be the names for the classes of software systems belonging to a certain family. Let $Z$ be the minimal total number of workplaces necessary for the plant, $P$ be the available financial resources, $d_{j}$ be the average price of one workplace for the system of the class $A_{j}$. It is required to find the $i$-th combination of the classes $\left\{A_{j}\right\}$ and the numbers of workplaces $m_{j i}$ in that combination, so that the following objective function would be maximized:

$$
Q_{i}=\left(E\left(C_{n i}^{k}\right) / D\left(C_{n i}^{k}\right)\right) \rightarrow \max ,
$$

where $Q_{i}$ is the efficiency degree for the software system per price unit, $C_{n i}^{k}$ is the $i$-th combination of classes containing $n$ classes of the system among $k$ possible classes (i.e. it is a certain set containing $n$ classes, and being a subset for the given set containing $k$ classes, $n \leq k), E\left(C_{n i}^{k}\right)$ is the functionality degree for the combination $C_{n i}^{k}$, and $D\left(C_{n i}^{k}\right)$ is price of this combination. The following requirements have to be satisfied as well: 1) The price of any combination must not exceed the available financial resources; 2) No combination may contain identical elements; 3) Each pair of combinations must differ in at least one element; 4) The whole set of combinations must contain all the unary, binary, ternary, etc. combinations of classes; 5) The total number of workplaces for all the classes forming any combination must not be less than minimally possible value $Z$.

The functionality degrees $E\left(C_{n i}^{k}\right)$ are evaluated using the AHP by calculating with the help of pairwise comparison matrix (PCM) the vector of priorities for all possible combinations of existing classes. The 
number $m_{j i}$ of workplaces of the $j$-th class in their $i$-th combination is evaluated using the AHP by calculating with the help of PCMs the vectors of priorities for various percentage ratios of workplaces numbers in each possible combination of classes.

The optimal solution for the above-formalized problem is found among all the combinations of classes by means of exhaustive search. Additionally, various percentage ratios of workplaces numbers (with respect to their priorities) are looked through while examining each binary, ternary, etc. combinations of classes. The search algorithm has the following steps:

1) The initial set of alternatives is formed. It must include all possible combinations of classes for the given family of systems. The total number of these combinations is:

$$
A=k ! /(n !(k-n) !) \text {. }
$$

For instance if the number of existing classes $k=3$ then there are the following combinations:

$$
C_{11}^{3}=A_{1}, C_{12}^{3}=A_{2}, C_{13}^{3}=A_{3}, C_{24}^{3}=A_{1} A_{2}, C_{25}^{3}=A_{1} A_{3}, C_{26}^{3}=A_{2} A_{3}, C_{37}^{3}=A_{1} A_{2} A_{3}
$$

2) The PCM for the obtained set of alternatives is formed, and the vector of priorities is evaluated:

$$
W_{n}^{k}=\left(w_{1}, \ldots, w_{A}\right),
$$

where $A$ is defined by (2). The elements $w_{i}$ of this vector are just the functionality degrees $E\left(C_{n i}^{k}\right)$ for the corresponding combinations of classes. For instance if $k=3$ then we will have:

$$
\begin{gathered}
E\left(C_{11}^{3}\right)=w_{1}, E\left(C_{12}^{3}\right)=w_{2}, E\left(C_{13}^{3}\right)=w_{3}, \\
E\left(C_{24}^{3}\right)=w_{4}, E\left(C_{25}^{3}\right)=w_{5}, E\left(C_{26}^{3}\right)=w_{6}, E\left(C_{27}^{3}\right)=w_{7} .
\end{gathered}
$$

3) The numbers $m_{j i}$ of workplaces is determined for each class in every combination, subject to the requirement 5 , i.e.

$$
\sum_{j=1}^{k} m_{j i} \geq Z ; i=1, \ldots, A .
$$

For each unary combination we can set $m_{j i}=Z$ if the $i$-th combination is represented by the class $A_{j}$, and $m_{j i}=0$ otherwise. In case of binary, ternary and other combinations we can evaluate $m_{j i}$ by AHP in the following way: The subset containing all the combinations except unary ones is separated out of the initial set of alternatives. The number of elements in that subset is $A-k$. Let $\alpha_{j i}$ be the relative number (percentage) of the workplaces for the class $A_{j}$ in the $i$-th combination. It is obvious that:

$$
\sum_{j=1}^{k} \alpha_{j i}=100 .
$$

A PCM is formed for every combination belonging to that subset. The object for comparison is the acceptability (for the plant) of various percentage ratios of workplaces numbers for different classes. For instance if $k=3$ then we have four matrices $(i=4 ; 5 ; 6 ; 7)$, and each of them is aimed at comparing acceptability of various values for the following kinds of ratios (respectively):

$$
\left(\alpha_{14} / \alpha_{24}\right)_{A_{1} A_{2}},\left(\alpha_{15} / \alpha_{35}\right)_{A_{1} A_{3}},\left(\alpha_{26} / \alpha_{36}\right)_{A_{2} A_{3}},\left(\alpha_{17} / \alpha_{27} / \alpha_{37}\right)_{A_{1} A_{2} A_{3}} .
$$

For example the expression $\left(\alpha_{14} / \alpha_{24}\right)_{A_{1} A_{2}}=10 / 90$ means that the combination $C_{24}^{3}=A_{1} A_{2}$ contains $10 \%$ workplaces of the class $A_{1}$ and $90 \%$ workplaces of the class $A_{2}$. Another example, the expression $\left(\alpha_{17} / \alpha_{27} / \alpha_{37}\right)_{A_{1} A_{2} A_{3}}=5 / 10 / 85$ means that the combination $C_{37}^{3}=A_{1} A_{2} A_{3}$ contains $5 \%$ workplaces of the class $A_{1}, 10 \%$ workplaces of the class $A_{2}$, and $85 \%$ workplaces of the class $A_{3}$.

The priority vectors corresponding to each of the described matrices are evaluated. The values of components for a certain vector determine preferences of different percentage ratios for numbers of workplaces of different classes in their particular combination. As a result, we can find the most preferable percent- 
age ratio for every combination. The corresponding absolute values $m_{j i}$ can be calculated using the formula:

$$
m_{j i}=r\left(Z \cdot \alpha_{j i} / 100\right),
$$

where $r(x)$ means rounding of $x$ to the nearest greater integer (this keeps the condition (6) satisfied).

4) At this step the obtained values $m_{j i}$ are checked for satisfying the requirement 5 connected with the total price of the system. In other words, the following condition is checked:

$$
D\left(C_{n i}^{k}\right)=\sum_{j=1}^{k} d_{j} m_{j i} \leq P ; i=1, \ldots, A .
$$

The set of feasible alternatives is formed as the result of that check. An alternative, represented by only one class, is considered to be feasible if it satisfies the condition (10); in this case the sum in (10) has only one non-zero component. An alternative, represented by binary, ternary, etc. combination of classes must be also checked for satisfying the condition (10), and if the alternative does not, the next percentage ratio (respect to decreasing of the priorities) is taken: the new values $m_{j i}$ are obtained using (9), and the corrected in this manner alternative is checked for satisfying (10) again, etc. It can be shown that the set of feasible alternatives is not empty if and only if the following condition holds:

$$
d_{*} \cdot Z \leq P,
$$

where $d_{*}$ is the minimal element in the set $\left\{d_{j} \mid j=1, \ldots, k\right\}$ (i.e. it is the price of the cheapest workplace).

5) Now we can find the optimal solution for the problem connected with choice of class of system. The optimal solution is found among the feasible solutions using the formula (1). The obtained solution should be drawn up as a specification containing the classes having been chosen and numbers of workplaces determined for each class.

\subsection{The choice of type of system}

We solve this problem by direct using of the AHP. The solving algorithm has the following steps:

1) The initial set of alternatives $\left\{A_{1}, A_{2}, \ldots, A_{n}\right\}$ is formed. Each alternative represents a certain type of software systems for the class having been chosen at the previous stage.

2) The hierarchy having several levels is constructed. The highest (nearest to the root) level contains a set of group criteria $\left\{Q_{1}, \ldots, Q_{K}\right\}$, the next one contains a set of criteria $\left\{Q_{11}, \ldots, Q_{1 m} ; \ldots ; Q_{K 1}, \ldots, Q_{K l}\right\}$ detailing the corresponding group criteria, etc. For instance, the following two-level hierarchy can be used for CAD/CAM/CAE systems of the "heavy" class: the higher level of this hierarchy contains nine generalzied criteria $(K=9)$ : $Q_{1}=$ "User Interface", $Q_{2}=$ "User Support", $Q_{3}=$ "Geometric Data Exchange", $Q_{4}=$ "Geometric Modelling", $Q_{5}=$ "Design Documentation", $Q_{6}=$ "Applied Tasks", $Q_{7}=$ "Programming for Numerical Control Machines", $Q_{8}=$ "Database Management System", $Q_{9}=$ "Reverse Engineering". A certain set of the particular criteria from the lower level corresponds to each of the abovementioned criteria from the higher level. For example, the criteria corresponding to $Q_{4}$ ("Geometric Modelling")

are:

$Q_{41}=$ "Skeleton Modelling", $Q_{42}=$ "Surface Modelling", $Q_{43}=$ "Solid-state Modelling", $Q_{44}=$ "Parametric Modelling", $Q_{45}=$ "Preliminary Designing", $Q_{46}=$ "Assembly Designing", $Q_{47}=$ "Associative Connections between Details".

3) The vectors of priorities for alternatives respect to the last but one hierarchy level are calculated by forming and processing the corresponding PCMs. The PCMs for remaining hierarchy levels are processed analogously, and the priorities for the elements of a certain hierarchy level respect to the higher level elements being connected with them are calculated. Thus, we get the set of all priority vectors generated by the existing hierarchy. 
4) The synthesis is executed, that means successive calculation of priorities for alternatives respect to the elements belonging to all the hierarchy levels. This process has the direction from the lower hierarchy levels to the higher ones, subject to the particular connection mode between elements of adjacent levels. The ultimate aim is evaluating the priorities of alternatives respect to the root element of the hierarchy.

\subsection{The choice of configuration for a workplace}

The developed by the authors mathematical model for this stage is based on the Combinatorial and Morphological Resources Allocation method (Andreichicov and Andreichicova, 2000). The model is underlain by the idea of using a morphological table, the structure of which is shown in Fig. 2. This table describes a set of possible configurations for a software system of a certain type in compact and clear form.

\begin{tabular}{|c|c|c|c|c|c|}
\hline \multicolumn{2}{|c|}{ Component parts for configurations } & \multirow{2}{*}{\multicolumn{4}{|c|}{$\begin{array}{c}\text { ALTERNATIVES } \\
\left(A_{i j}\right)\end{array}$}} \\
\hline Names & Specifications & & & & \\
\hline \multirow{4}{*}{ Part 1} & Alternatives $\left(A_{i j}\right)$ & $A_{11}$ & $A_{12}$ & .. & $A_{1 k 1}$ \\
\hline & Efficiency $\left(E_{i j}\right)$ & $E_{11}$ & $E_{12}$ & $\ldots$ & $E_{1 \mathrm{k} 1}$ \\
\hline & Price $\left(d_{i j}\right)$ & $d_{11}$ & $d_{12}=$ & $\ddot{\ddot{\theta}}$ & $d_{1 k 1}$ \\
\hline & Quantity $\left(z_{i j}\right)$ & $z_{11}$ & $z_{12}$ & $\ldots$ & $z_{1 k 1}$ \\
\hline \multirow{4}{*}{ Part 2} & Alternatives $\left(A_{i j}\right)$ & $A_{21}$ & $A_{22}$ & $\ldots$ & $A_{2 \mathrm{k} 2}$ \\
\hline & Efficiency $\left(E_{i j}\right)$ & $E_{21}$ & $E_{22}$ & $\ldots$ & $E_{2 k 2}$ \\
\hline & Price $\left(d_{i j}\right)$ & $d_{21}$ & $d_{22}$ & & $d_{2 k 2}$ \\
\hline & Quantity $\left(z_{i j}\right)$ & $z_{21}$ & $z_{22}$ & $\ldots$ & $z_{2 k 2}$ \\
\hline & 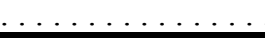 & . & & . & \\
\hline \multirow{4}{*}{ Part $n$} & Alternatives $\left(A_{i j}\right)$ & $A_{n 1}$ & $A_{n 2}$ & .. & $A_{n \mathrm{~km}}$ \\
\hline & Efficiency $\left(E_{i j}\right)$ & $E_{n 1}$ & $E_{n 2}$ & $\ldots$ & $E_{n \mathrm{~km}}$ \\
\hline & Price $\left(d_{i j}\right)$ & $d_{n 1}$ & $d_{n 2}$ & $\ldots$ & $d_{n \mathrm{~km}}$ \\
\hline & Quantity $\left(z_{i j}\right)$ & $z_{n 1}$ & $z_{n 2}$ &. & $z_{n \mathrm{~km}}$ \\
\hline
\end{tabular}

Fig. 2. The structure for morphological table of configurations

According to Fig.2, a workplace configuration has the following structure:

1) There is a set of component parts (subsystems, units, details, modules, packages, etc.), determined by their names. Let $n$ be the total number of the available component parts.

2) Each component part is determined by three sets of specifications: efficiency $\left(E_{i j}\right)$, price $\left(d_{i j}\right)$ and quantity $\left(z_{i j}\right)$. The efficiency is evaluated by the AHP similarly to the efficiency evaluating for the types of system (see 2.3). The sources of information about the prices are vendors' price-lists. The quantity is determined by the functional characteristics of the systems.

3) Each component part is realized by a finite set of alternatives $A_{i j}$. The maximum value $\left(k_{1}, k_{2}, \ldots, k_{m}\right)$ of the column index $j$ here is different for each row $i$.

Thus, the configuration choice problem for the software system of a certain type can be formalized in the following way. Assume the morphological table of configurations has been set (with the similar structure to the one shown in Fig. 2). Let $R$ be the highest allowed price for configurations. It is required to find a combination $\left\{A_{i j}\right\}$ of alternatives, so that the following objective function would be maximized:

$$
Q=\left(\sum_{i=1}^{n} E_{i j(i)} / \sum_{i=1}^{n} d_{i j(i)} \cdot z_{i j(i)}\right) \rightarrow \max .
$$

The following requirements have to be satisfied here:

1) The price of any combination must not exceed the specified upper limit, i.e. 


$$
\sum_{i=1}^{n} d_{i j(i)} \cdot z_{i j(i)} \leq R
$$

2) Each combination must include an alternative $A_{i j}$ from every row of the morphological table respect to the specified quantity $z_{i j}$. This requirement is rather strong and in some situations it may be weakened. For example, in case of limited financial resources it may be sometimes impossible to allocate the latter among all the rows of the morphological table. In such cases the following requirement should be used instead of the current one: 2 a) Each combination should include an alternative from a certain subset of the morphological table rows respect to the specified quantity $z_{i j}$.

The algorithm for the specified configuration choice problem has the following steps: 1) Form the morphological table; 2) Specify the highest allowed price for configurations; 3) Form the set of all possible combinations of alternatives, using exhaustive search, respect to the condition 2 or 2a; 4) Calculate the total price for each obtained combination, and check if the condition (13) is satisfied. Thus, the set of feasible solutions is formed; 5) Find the optimal solution among the feasible ones using the formula (12). The obtained structure of optimal configuration should be drawn up as a specification to a contract for the sale of software system of the chosen type.

\section{The choice of optimal decision in fuzzy environment}

The above-described models and algorithms for optimal decision choice correspond to the case when all the initial data for the choice problem are well-defined and exactly known. But this usually does not occur. First, limits for price of software system and for number of workplaces are usually not strict enough - the sets of allowed values for those parameters have usually vague bounds; the last fact may have interpretation of different preference levels for different values. Second, values of many parameters characterizing a software system are often uncertain (the average price of workplace is good example here). The information about those values is usually available in the form of expert judgements, which are often expressed in linguistic form. Thus, the initial mathematical model for the choice problem has to be adapted for taking into account uncertainty and ambiguity of initial information. The mathematical conception of fuzzy sets, fuzzy numbers and linguistic variables (Zadeh, 1973) seems to be the most effective here.

We will view here the way for fuzzification of the model for choice of class of system, described in 3.1. For the sake of simplicity we will assume that only the desired price of a software system $P$ and the average price of workplace $d_{j}$ (see 3.1) are fuzzy. Additionally, let the values of the parameter $d_{j}$ be estimated by experts in linguistic form using expressions like these: "high", "medium", "low", "not very high", "more or less low", etc. We will use fuzzy numbers or fuzzy intervals generalizing them (Dubois and Prade, 1979) for formalization of fuzzy values. According to its definition, fuzzy interval is fuzzy subset of the real line with membership function having the following structure:

$$
\mu_{C}(x)=\left\{\begin{array}{l}
L_{C}(x), a_{0} \leq x \leq a_{1}, \\
1, a_{1}<x<b_{1} \\
R_{C}(x), b_{1} \leq x \leq b_{0}, \\
0, \text { otherwise }
\end{array}\right.
$$

where $L_{C}, R_{C}$ are piecewise-continuos functions such that $L_{C}$ increases in the segment $\left[a_{0}, a_{1}\right]$ from $L_{C}\left(a_{0}\right)=0$ to $L_{C}\left(a_{1}\right)=1$, and $R_{C}$ decreases in the segment $\left[b_{1}, b_{0}\right]$ from $R_{C}\left(b_{1}\right)=1$ to $R_{C}\left(b_{0}\right)=0$. If $a_{1}=b_{1}$ then $C$ is fuzzy number. The functions $L_{C}$ and $R_{C}$ are called left and right branches of the membership function of fuzzy interval $C$ respectively. In some cases one branch may be missing (i.e. may be $a_{0}=a_{1}$ or $\left.b_{1}=b_{0}\right)$.

Assume the following notation. Let $R^{+}$be the set of $(0, \propto), U=\left[u_{*}, u^{*}\right] \subset R^{+}$, where $u_{*}, u^{*}$ are respectively left and right bounds for the set of all possible prices of the workplaces for a software system of a 
certain type. We will formalize the values of $d_{j}$ using linguistic variable, and we will define the latter using the conventional structure (Zadeh, 1973):

$$
<L, T(L), U, G, M>\text {, }
$$

where $L$ is the name of variable, $T(L)$ is the set of names of its primary linguistic values (terms) with each value being a fuzzy interval defined on the universal set $U, G$ is a syntactic rule for generating the complex terms, and $M$ is a semantic rule for formalizing the latter. In our case $L=$ "Price of Workplace"; $T(L)=\{$ "low", "medium", "high" $\}$; the terms are represented in the form of fuzzy intervals on the above-defined set $U \subset R^{+}$. The model for membership functions of the primary terms is shown in Fig. 3.

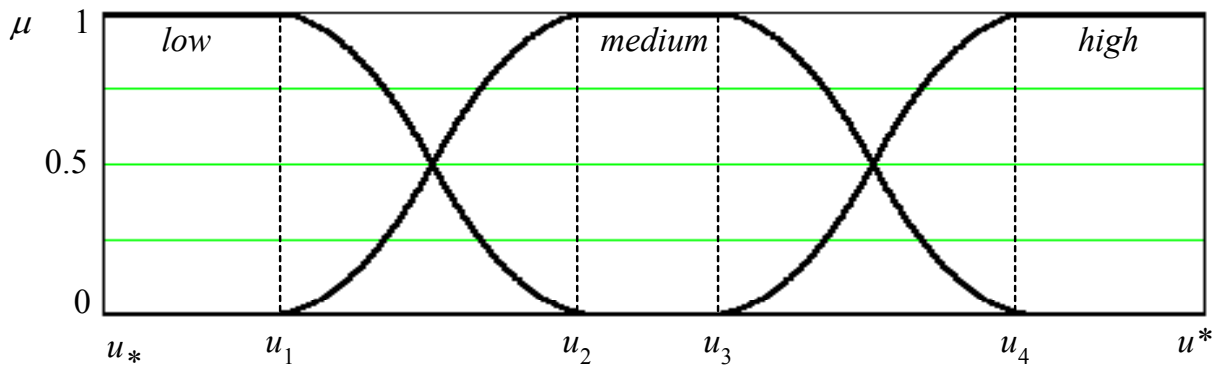

Fig. 3. The primary terms of the linguistic variable "Price of Workplace"

The values for $u_{1}, u_{2}, u_{3}, u_{4}$, and particular form for the branches of the membership functions should be set by experts on the basis of existing information on prices of workplaces for the software system being chosen, taking into account some known from literature recommendations on membership functions constructing. The syntactic rule $G$ allows to generate complex terms subject to the syntactic diagram shown in Fig. 4. The semantic rule $M$ associates concentrating (squaring) with the "very" modifier, dilatation (square rooting) with the "more or less" modifier, and complement with the "not" modifier. It can be shown that under these conditions any complex term will keep the property of being fuzzy interval. So, $d_{j} \in G[T(L)]$, where $G[T(L)]$ is the extended set of terms for the linguistic variable $L$, containing all possible values of it, and each value is represented by a fuzzy interval on the set $U$.

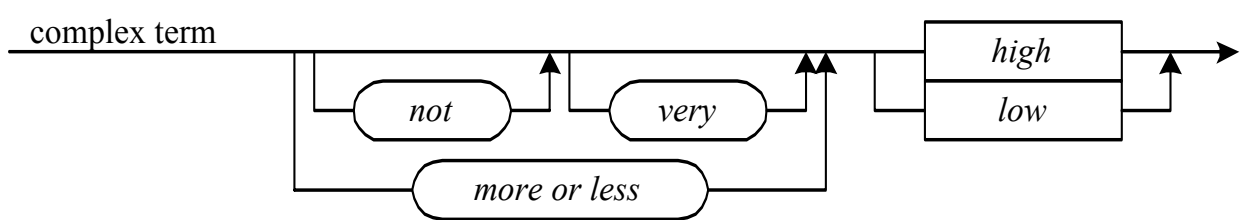

Fig. 4. Syntactic diagram for generating the complex terms

A fuzzy interval on the set $R^{+}$is used for representation of the desired price $P$. The membership function has the structure similar to (14). The degree of membership for a certain value to that interval may be interpreted as degree of acceptability of the corresponding price for the plant. Thus, the prices within the limits of $\left[a_{1}, b_{1}\right]$ are fully acceptable, and the prices out of the limits of $\left[a_{0}, b_{0}\right]$ are fully inadmissible.

Because of fuzziness of some initial parameters of the problem, the algorithm for its solving (searching for optimal combinations of the classes of system) described in 3.1 is modified as well. Particularly, the 4-th and 5-th steps are modified, and instead of them we have the following steps:

4a) The price for an $i$-th combination of classes is calculated by the following formula:

$$
D\left(C_{n i}^{k}\right)=\sum_{j=1}^{k} d_{j} m_{j i} .
$$

Since the values of $d_{j}$ are fuzzy, the using of fuzzy arithmetic (Dubois and Prade, 1979) is necessary in (16). Thus, the value of the price $D\left(C_{n i}^{k}\right)$ becomes a fuzzy interval as well. The obtained price $D\left(C_{n i}^{k}\right)$ is compared with the desired price $P$ in order to evaluate the acceptability degree for the combination 
$C_{n i}^{k}$ with respect to price. The concept for inclusion indices of fuzzy sets (Fuzzy Sets, 1982) can be used here. Assume $A$ and $B$ are arbitrary fuzzy sets on a set $X$. We take the following index, which evaluates the inclusion degree of $A$ into $B$.

$$
I(A, B)=|A \cap B| /|A|,
$$

where $|A|=\int_{X} \mu_{A}(x) d x$, and the used definition for fuzzy intersection is product-oriented (Zadeh, 1973). We should note the following properties of the above-defined index (Fuzzy Sets, 1982): a) $0 \leq I(A$, $B) \leq 1$; b) $I(A, B)=0$ if and only if supp $A \cap \operatorname{supp} B=\varnothing$; c) $I(A, B)=1$ if and only if $A \subseteq B_{1}$, where $B_{1}$ is the core of the set $B$ (i.e. the set of elements which membership degrees are 1); d) if $C \subseteq A$ then $I(C, B) \leq I(A$, $B)$. Due to these properties we can make the following definition for the acceptability degree for an alternative with respect to price:

$$
F\left(C_{n i}^{k}\right)=I\left(D\left(C_{n i}^{k}\right), P\right)
$$

The alternative is considered to be feasible if $F\left(C_{n i}^{k}\right)>0$. Otherwise, as it was in crisp case, the next percentage ratio (respect to decreasing of the priorities) is taken, and the operations are repeated. It can be shown that the set of feasible alternatives is not empty if and only if the following is true:

$$
\left[Z \cdot u_{*}, Z \cdot u^{*}\right] \cap \operatorname{supp} P \neq \varnothing \text {. }
$$

5a) Unlike in crisp case, we have the acceptability degree for combination of classes with respect to price instead of its price. Therefore, we cannot use directly the objective function (1) for evaluating the efficiency of the feasible alternatives. But if we treat efficiency as a generalized criterion being detailed by the criteria of funblctionality and acceptability with respect to price, we can use the AHP for efficiency evaluation. In other words, we can construct the hierarchy with these two criteria. We do not need to calculate the priorities of alternatives respect to these criteria, because we already have them: the values of $E\left(C_{n i}^{k}\right)$ have been calculated on the step 2, and the values of $F\left(C_{n i}^{k}\right)$ have been calculated on the step 4a.

\section{Conclusion}

Using of the AHP in combination with elements of fuzzy sets theory enables to formalize and effectively solve the complicated, multi-objective and ill-defined problem of optimal software system configuration choice and therefore decrease the mistaken decisions and the sequent significant financial loses risk level. The developed by authors mathematical models and algorithms were tested on different tasks connected with optimal CAD/CAM/CAE systems configuration choice (Avertchenkov, Miroshnikov and Podvessovski, 1999). The expert system for automation of choosing optimal CAD/CAM/CAE systems configurations for engineering plants was developed on the basis of those models and algorithms.

\section{References}

Andreichicov, A.V. and Andreichicova, O.N. (2000), Decision Analysis, Synthesis and Planning in Economics, Finansy i Statistika, Moscow (in Russian).

Avertchenkov, V., Miroshnikov, V. and Podvessovski, A. (1999), "The Methods of Knowledge Representation in the Expert System for CAD/CAM/CAE System Optimal Configuration Choice", Proceedings of the Sixth International Conference on Advanced Computer Systems (ACS' 99), Informa, Szczecin, Poland, 431-434.

Dubois, D. and Prade, H. (1979), "Fuzzy Real Algebra: Some Results", Fuzzy Sets and Systems, 2, 327348. 
Fuzzy Sets and Possibility Theory: Recent Developments (1982), R.R. Yager eds., Pergamon Press, New York.

Saaty, T.L. (1980), The Analytic Hierarchy Process: Planning, Priority Setting, Resource Allocation, McGraw-Hill, New York.

Zadeh, L.A. (1973), The Concept of a Linguistic Variable and Its Application to Approximate Reasoning, American Elsevier Publishing Company, New York. 
